\title{
High-risk Endometrial Cancer: The Present and Future of Adjuvant Therapy
}

\author{
Whitney Soble, ${ }^{1}$ Joan Tymon-Rosario ${ }^{1}$ and Gloria S Huang ${ }^{1,2}$
}

1. Department of Obstetrics, Gynecology and Reproductive Sciences, Yale School of Medicine, New Haven, CT, USA; 2. Yale Cancer Center, Yale School of Medicine, New Haven, CT, USA

DOI: https://doi.org/10.17925/OHR.2021.17.1.23

$\square$ ndometrial cancer is the most common invasive gynaecologic malignancy in developed countries. High-risk endometrial cancer is a heterogenous subgroup with a more aggressive clinical course. It includes those with one or more defined clinical or pathological risk factors for recurrence and mortality. Surgery remains the primary treatment modality for women with resectable endometrial cancer. However, the optimal adjuvant therapy following surgical resection and staging remains an area of active investigation. Here, we review the current treatment landscape for women with high-risk endometrial cancer, with a focus on the most recently published literature. Several high-quality, randomized clinical trials, including GOG-249 and GOG-258, were recently completed to address key questions in the management of high-risk endometrial cancer. The evidence confirms the role of radiation therapy in patients with high-risk, early-stage disease. For patients with advanced-stage disease, systemic chemotherapy is indicated to reduce the risk of distant metastasis. Ongoing clinical trials address the emerging role of immunotherapy in endometrial cancer, and its potential utility in the adjuvant setting.

\section{Keywords}

Endometrial cancer, high-risk, advanced stage, adjuvant treatment, chemotherapy, radiotherapy

Disclosure: Gloria S Huang has received consulting fees from Bristol Myers Squibb, Tesaro and AstraZeneca. Whitney Soble and Joan Tymon-Rosario have no financial or non-financial relationships or activities to declare in relation to this article.

Review process: Double-blind peer review.

Compliance with ethics: This study involves a review of the literature and did not involve any studies with human or animal subjects performed by any of the authors.

Authorship: The named authors meet the International Committee of Medical Journal Editors (ICMJE) criteria for authorship of this manuscript, take responsibility for the integrity of the work as a whole, and have given final approval for the version to be published.

Access: This article is freely accessible at touchONCOLOGY.com (c) Touch Medical Media 2021

Received: 26 August 2020

Accepted: 10 February 2021

Published online: 27 July 2021

Citation: touchREVIEWS in Oncology \& Haematology. 2021;17(1):23-7

Corresponding author: Gloria Huang, Department of Obstetrics, Gynecology and Reproductive Sciences,

PO Box 208063, Yale School of Medicine, New Haven,

CT 06520-8063, USA.

E: gloria.huang@yale.edu

Support: No funding was received for

the publication of this article.
Endometrial cancer has become an increasingly significant cause of morbidity and mortality for women. Currently, endometrial cancer is the most common invasive gynaecologic malignancy, and the ninth most common cancer among women in the USA, accounting for approximately 65,620 new cancer cases and 12,590 deaths in $2020 .{ }^{1}$ The 5 -year relative survival rate is estimated to be $83 \% .{ }^{2}$ However, these rates vary drastically across cancer stage and histologic subtype, from $95 \%$ for patients with localized disease compared with $<20 \%$ among women with distant spread. Endometrial deaths have increased steadily by approximately $20 \%$ over the past decade. If current trends continue, the incidence of endometrial cancer is expected to double by 2030, and deaths from endometrial cancer will soon surpass those from ovarian cancer. ${ }^{3}$

Traditionally, endometrial cancer has been subdivided according to a histopathologic paradigm. ${ }^{4}$ Type I endometrial cancer includes grade 1 and 2 endometrioid endometrial adenocarcinoma. Unopposed oestrogen and hyperinsulinaemia increase the risk of developing these cancers, and these signalling pathways are linked to clinical risk factors, such as obesity, diabetes and polycystic ovarian syndrome. The precursor lesion for these malignancies is atypical endometrial hyperplasia. Patients typically present with early-stage disease and have a favourable prognosis. Type II endometrial cancers comprise a heterogenous group of endometrial histologic subtypes, including grade 3 endometrioid endometrial adenocarcinoma, as well as papillary serous, clear cell and undifferentiated carcinomas. The oncogenic pathways and precursor lesions related to the development of these aggressive malignancies are not yet well defined.

For all histologic subtypes of endometrial cancer, standard initial treatment is surgical, with total hysterectomy and bilateral salpingo-oophorectomy. Surgical staging aids in both prognostic stratification and identification of patients who may benefit from adjuvant therapy. Pelvic and para-aortic lymphadenectomy is controversial, given its uncertain therapeutic role and risk for subsequent lymphedema, and in recent years sentinel lymph node biopsy has gained favour among many surgeons. ${ }^{5}$ Omentectomy is frequently performed for serous or clear-cell histology, for cytoreductive purposes, or for detection of occult metastases. Chemotherapy, radiation, endocrine therapy or combination treatments may be pursued in patients deemed to be poor surgical candidates, or those presenting with widely metastatic and unresectable disease. In this review, we focus on the adjuvant treatment of women who have undergone initial surgical management for high-risk endometrial cancer, with a focus on the recently published clinical trials in this area.

\section{Early-stage, high-risk endometrial cancer}

Historically, patients with high-risk, early-stage disease were treated with adjuvant external beam radiation therapy (EBRT), with the aim of decreasing the incidence of recurrence. This practice was controversial in some early-stage endometrial cancers, in which the risk of recurrence might 
be expected to be low, even in the absence of adjuvant therapy. The Gynecologic Oncology Group (GOG), therefore, set out to compare progression-free survival (PFS) and overall survival (OS) in patients with stage I/II endometrial cancer, randomized to either pelvic EBRT or no adjuvant therapy in GOG 99.6 Of note, serous and clear-cell histology were excluded given their greater propensity for recurrence. This was, therefore, a study of intermediate-risk disease. Patients fulfilling these inclusion criteria were noted to have a largely favourable outcome, with a 2-year recurrence rate of $12 \%$ among those randomized to receive no adjuvant treatment. Participants were, therefore, stratified into lowintermediate and high-intermediate risk categories according to risk factors, including histologic grade 2 or 3, deep myometrial invasion and presence of lymphovascular space invasion. Subjects were determined to have high-intermediate risk disease if they were aged 70 or older with one risk factor, aged 50-69 with two risk factors, or aged 18 or older with all three risk factors.

One-third of the study population qualified as high-intermediate risk and demonstrated a 2-year recurrence rate of $27 \%$ among patients who did not receive adjuvant therapy, compared with a rate of $6 \%$ among untreated patients in the low-intermediate risk group. Adjuvant pelvic EBRT was confirmed to significantly reduce the rate of local recurrence among both high-intermediate risk (hazard ratio [HR] 0.58) and lowintermediate risk (HR 0.54) groups, albeit with a greater absolute treatment effect in the high-intermediate risk group (4-year recurrence rate of $13 \%$ versus $27 \%$ in treated versus untreated patients in the highintermediate risk group, compared with $2 \%$ versus $6 \%$, respectively, in the low-intermediate risk group). Based on these findings, adjuvant pelvic EBRT appears to decrease the risk of recurrence, but treatment should be restricted to patients who meet the criteria for high-intermediate risk disease.

These findings were concordant with the results of PORTEC-1, in which patients with low- and intermediate-risk endometrial cancer were found to have significantly decreased risk of locoregional recurrence with adjuvant pelvic radiotherapy compared with no adjuvant therapy (5\% versus $14 \%$ ) without any concurrent survival benefit (81\% versus $85 \%$ ).? The results of these landmark trials led to a significant decrease in the use of adjuvant radiotherapy and a concomitant reduction in radiotherapyrelated morbidity for women with low-intermediate risk disease. ${ }^{8}$ For women with high-intermediate risk disease receiving adjuvant pelvic EBRT, planning and delivery techniques should ensure that the vaginal cuff and all locoregionally-draining lymphatics are included in the treatment fields.

It was noted in GOG 99 that, among patients who experienced recurrence, a common site of this was the vaginal cuff. This raised the question of whether vaginal brachytherapy might offer similar reduction in recurrence risk while decreasing the toxicity associated with treatment. This was investigated in PORTEC-2, in which patients with high-intermediate risk disease were treated with either vaginal brachytherapy or pelvic EBRT. This study demonstrated equivalent locoregional recurrence rates but a favourable toxicity profile with vaginal brachytherapy compared with pelvic EBRT. ${ }^{9}$ It is worth noting that this study used a different definition of 'high-intermediate risk' than that used in GOG 99 (age greater than 60 years with stage IB grade 3 disease, or stage IC grade 1 or 2 disease, or stage IIA disease at any age) and participants were, therefore, likely to be at a lower overall risk of recurrence compared with the participants in the high-intermediate risk group in the earlier study. To date, there have been no equivalent studies comparing vaginal brachytherapy and EBRT in early-stage high-risk endometrial cancer. Nevertheless, based on these findings, adjuvant vaginal brachytherapy has become the standard of care for the treatment of high-intermediate risk endometrial cancer for optimization of vaginal disease control with minimal morbidity.

As radiation therapy reduces the risk of locoregional recurrence but does not address the risk of distant recurrence, there is interest in the use of a combined approach of chemotherapy plus some form of radiotherapy. This was addressed in GOG 249, in which patients with high-intermediate risk and high-risk early-stage disease were randomized to receive either vaginal brachytherapy followed by three cycles of chemotherapy, or pelvic EBRT. ${ }^{10}$ Specific high-risk inclusion criteria included stage II endometrioid histology and stage I/II serous or clear-cell histology. Patients in the vaginal brachytherapy/chemotherapy arm received either high-dose brachytherapy (600-700 cGy at a depth of $0.5 \mathrm{~cm}$ for three fractions, 1,000-1,050 cGy at the vaginal surface for three fractions or $600 \mathrm{cGy}$ at the vaginal surface for five fractions) or low-dose brachytherapy (6,500-7,000 cGy at the vaginal surface in one to two insertions at a dose rate of 400-1,000 cGy per hour), followed by chemotherapy consisting of paclitaxel $\left(175 \mathrm{mg} / \mathrm{m}^{2}\right)$ and carboplatin (area under curve $[\mathrm{AUC}] \mathrm{6}$ ) every 21 days for a total of three cycles. Patients in the pelvic EBRT arm received 4,500-5,040 cGy over 5-6 weeks (180 cGy per day for 25-28 fractions), and patients with cervical involvement or serous or clear-cell histology were permitted to receive brachytherapy boosts.

At a median follow-up of 53 months, there were no statistically significant increases in 5-year PFS or OS in the vaginal brachytherapy/ chemotherapy arm compared with the pelvic EBRT arm (PFS: HR 0.92, 90\% confidence interval [CI] 0.69-1.23; OS: HR 1.04, 90\% Cl 0.71-1.52). However, the 5-year cumulative incidence of pelvic or para-aortic node recurrence was more than twice as high in the vaginal brachytherapy/ chemotherapy group compared to the pelvic EBRT group ( $9 \%$ versus $4 \%$; HR $0.47,95 \% \mathrm{Cl} 0.24-0.94)$. Regarding other types of recurrences, distant recurrence was much more common than vaginal recurrence $(18 \%$ versus 2.5\%), but there was no significant difference between the groups with respect to either vaginal recurrence or distant recurrence (HR 1.0 for each). Completion rates were high (91\% in the radiotherapy group, 32\% of whom also received vaginal brachytherapy, and $87 \%$ in the vaginal brachytherapy/chemotherapy group) and recurrence during treatment was rare $(0.3 \%$ in the radiotherapy group and $1.3 \%$ in the vaginal brachytherapy/chemotherapy group). Of note, most patients had stage I disease (74\%) and endometrioid histology (71\% versus $15 \%$ serous and $5 \%$ clear cell), an important consideration regarding the generalisability of the findings. Nevertheless, GOG 249 affirmed the role of radiation in the adjuvant treatment of patients with early-stage high-risk endometrial cancer for local disease control.

\section{Advanced-stage high-risk endometrial cancer}

Due to the heightened risk of distant recurrence in patients presenting with advanced disease, systemic therapy is now considered of paramount importance in the management of these patients. Traditionally, wholeabdomen radiation was the standard of care for adjuvant therapy in patients with stage III or IV endometrial cancer. In 2006, GOG 122 randomized patients with stage III or IV endometrial cancer of any histology and no more than $2 \mathrm{~cm}$ of postoperative residual disease to receive either whole-abdomen radiation (3,000 cGy in 20 daily fractions with a 15 cGy boost to the pelvic and para-aortic lymph nodes) or chemotherapy (doxorubicin $60 \mathrm{mg} / \mathrm{m}^{2}$ plus cisplatin $50 \mathrm{mg} / \mathrm{m}^{2}$ every 21 days for seven cycles, followed by one additional cycle of cisplatin only). ${ }^{11}$ The chemotherapy arm demonstrated significantly improved PFS and OS compared with the radiotherapy arm (PFS: HR 0.71, p=0.007; OS: HR 0.68, 
$p=0.004)$. This study established the benefits of adjuvant chemotherapy for treatment of locally advanced disease.

In GOG 122, initial pelvic recurrences were reported in 18\% of patients in the chemotherapy arm and $13 \%$ of patients in the radiotherapy arm, while initial abdominal recurrences outside of the pelvis were noted in $32 \%$ and $38 \%$, respectively. Local recurrence therefore continues to be a risk in patients receiving chemotherapy, and distant recurrence remains a problem in those receiving radiotherapy treatment. Given these outcomes, a combined approach utilising both chemotherapy and radiotherapy has been explored in this patient population. RTOG 9708 was a phase II study assessing feasibility, safety, toxicity, and overall and PFS in patients receiving adjuvant chemotherapy combined with radiation for treatment of high-risk endometrial cancer. ${ }^{12}$

The patient population for this study was selected to represent a group at increased risk of pelvic recurrence that might benefit from radiotherapy, as well as with evidence of distant dissemination that might benefit from chemotherapy. Inclusion criteria included grade 2 or 3 endometrioid histology with $>50 \%$ myometrial invasion (stage IB), stromal invasion of the cervix (stage II), or extrauterine disease confined to the pelvis (stage IIIA-C1). Serous and clear-cell histology were excluded. Patients received a total of 4,500 cGy of pelvic EBRT over 5 weeks in daily fractions of $180 \mathrm{cGy}$, with cisplatin $\left(50 \mathrm{mg} / \mathrm{m}^{2}\right)$ administered on days 1 and 21 of radiotherapy. This was followed by vaginal brachytherapy in the form of a single low-dose rate application of 2,000 cGy to the vaginal surface or three high-dose rate applications totalling 1800 cGy to the vaginal surface, followed by four additional courses of cisplatin and paclitaxel $\left(175 \mathrm{mg} / \mathrm{m}^{2}\right)$ every 28 days for four cycles. OS across participants in the study was $85 \%$ and PFS was $81 \%$. Recurrence rates were $2 \%$ at pelvic sites, $2 \%$ at regional sites, and $19 \%$ at distant sites. This represents an improvement in both pelvic and distant recurrence rates compared with GOG 122. ${ }^{12}$

Similarly, GOG 184 compared two chemotherapy regimens after tumour volume-directed EBRT. ${ }^{13}$ The study population included patients with stage III or IVA endometrial carcinoma of any histology, including serous and clear cell, with no more than $2 \mathrm{~cm}$ of residual postoperative disease. Patients were randomised to receive doxorubicin $\left(45 \mathrm{mg} / \mathrm{m}^{2}\right)$ followed by cisplatin $\left(50 \mathrm{mg} / \mathrm{m}^{2}\right)$ every 21 days for six cycles, with or without paclitaxel $\left(160 \mathrm{mg} / \mathrm{m}^{2}\right)$ on day 2 of each cycle. Patients in both arms were first treated with pelvic EBRT for a total dose of 5,040 cGy, administered in $180 \mathrm{cGy}$ fractions, with an optional intravaginal boost. Recurrence-free survival at 3 years was $62 \%$ in the cisplatin-doxorubicin arm and $64 \%$ in the cisplatin-doxorubicin-paclitaxel arm (HR 0.90, $95 \% \mathrm{Cl}$ 0.69-1.17). While addition of paclitaxel to the combination of doxorubicin and cisplatin failed to improve PFS and was associated with increased neurotoxicity, approximately $80 \%$ of patients completed the full six cycles of their assigned chemotherapy regimen, confirming the feasibility of chemotherapy administration following a course of radiation. Taken together, these study results support the feasibility and efficacy of a combined treatment strategy, but its efficacy relative to that of chemotherapy alone remained to be investigated.

This question was addressed by GOG 258 , a phase III trial that sought to investigate whether treatment with combination chemoradiotherapy is associated with longer PFS compared to chemotherapy alone for patients with stage III or IVA endometrial cancer. ${ }^{14}$ Eligibility criteria included stage III or IVA endometrial carcinoma of any histologic subtype, or stage I or II serous or clear-cell endometrial carcinoma with positive peritoneal washings. Almost $95 \%$ of patients had stage III disease, and patients with residual tumour $>2 \mathrm{~cm}$ were excluded. A total of 736 patients were eligible for analysis, of whom 346 received chemoradiotherapy and 361 received chemotherapy only. The chemoradiotherapy arm received cisplatin $\left(50 \mathrm{mg} / \mathrm{m}^{2}\right)$ on days 1 and 28 concurrent with tumour volumedirected EBRT (total 4,500 cGy in 25 fractions at 180 cGy per fraction), followed by carboplatin (AUC 5-6) plus paclitaxel (175 mg/m²) every 21 days for four cycles. The chemotherapy-only arm received carboplatin (AUC 6) plus paclitaxel $\left(175 \mathrm{mg} / \mathrm{m}^{2}\right)$ every 21 days for six cycles.

Recurrence-free survival at 5 years was $59 \%$ in the chemoradiotherapy group and $58 \%$ in the chemotherapy-only group (HR 0.9, 90\% Cl $0.74-$ 1.10); thus, chemoradiotherapy failed to demonstrate superiority to chemotherapy only. An unplanned subgroup analysis of PFS did not identify a subgroup of patients who might have benefited more from chemoradiotherapy than from chemotherapy alone when stratified by age, histologic subtype, surgical stage, body mass index, or the presence or absence of gross residual disease. The cumulative incidence of vaginal recurrence at 5 years was $2 \%$ in the chemoradiotherapy group and $7 \%$ in the chemotherapy-only group (HR 0.36, 95\% Cl 0.16-0.82); thus, vaginal recurrence was lower in the chemoradiotherapy arm of the trial. Similarly, the cumulative incidence of pelvic or para-aortic node recurrence at 5 years was 11\% in the chemoradiotherapy group and 20\% in the chemotherapy-only group ( $\mathrm{HR} 0.43,95 \% \mathrm{Cl} 0.28-0.66$ ); thus, pelvic or para-aortic node recurrence was also lower in the chemoradiotherapy arm of the trial. ${ }^{14}$

Despite the finding that the addition of radiotherapy to chemotherapy caused a significant decrease in the incidence of recurrence in the vaginal cuff and the pelvic and para-aortic lymph nodes, the overall risk of progression remained similar between the two groups because the risk of distant recurrence was higher in the chemoradiotherapy arm, although this was on the cusp of statistical significance (27\% versus $21 \%$, HR 1.36, 95\% Cl 1.00-1.86). These findings do not appear to be related to the difference in the number of patients in each arm who completed therapy as prescribed; in the chemoradiation arm, $85 \%$ of patients received two cycles of cisplatin co-administered with radiation, and $75 \%$ of patients received all four planned cycles of carboplatin and paclitaxel, while in the chemotherapy-only group, $85 \%$ of patients received all six cycles during a median period of 17 weeks. Data on OS are not yet available for this trial.

Additional data on combination adjuvant treatment in this patient population comes from PORTEC-3. ${ }^{15}$ This trial sought to investigate whether treatment of high-risk endometrial cancer with combination chemoradiotherapy is associated with longer progression-free survival or OS compared to radiotherapy alone. Study eligibility criteria included stage IA grade 3 endometrioid carcinoma with lymphovascular space invasion, stage IB grade 3 endometrioid carcinoma, stage II or III endometrioid carcinoma (any grade), or stage I-III carcinoma with serous or clear-cell histology. Patients with any macroscopic residual disease were excluded. The study comprised $30 \%$ stage I disease, $25 \%$ stage II disease, and $45 \%$ stage III disease. Of 660 patients, 330 received chemoradiotherapy and 330 received radiotherapy only. The chemoradiotherapy group received EBRT for a total 4,860 cGy in 27 fractions of 180 cGy per fraction, along with two cycles of cisplatin (50 $\left.\mathrm{mg} / \mathrm{m}^{2}\right)$ in the first and fourth week of EBRT administration, followed by four cycles of carboplatin (AUC 5) and paclitaxel (175 mg/m²) every 21 days for four cycles. The radiotherapy-only group received EBRT for a total 4860 cGy in 27 fractions of 180 cGy per fraction. Vaginal brachytherapy was added for all patients with cervical invasion on pathology review, as well as per physician discretion. 
At 5 years, OS was $81.8 \%$ in the chemoradiotherapy group, compared with $76.7 \%$ in the radiotherapy-only group (HR $0.76,95 \% \mathrm{Cl} 0.54-1.06$ ). Thus, chemoradiotherapy approached, but failed to demonstrate, superiority to radiotherapy only. However, with respect to PFS, the chemoradiotherapy arm fared better than the radiotherapy-only arm (75.5\% versus 68.6\% [HR $0.71,95 \% \mathrm{Cl} 0.53-0.95]) .{ }^{15}$ Furthermore, a post-hoc analysis performed after 1 additional year of follow-up, to examine survival rates and patterns of recurrence, found significant improvements in both OS (HR 0.70, 95\% Cl 0.51-0.97) and PFS (HR 0.70, 95\% Cl 0.52-0.94) in the chemoradiotherapy group compared with the radiotherapy-only group, with the greatest absolute benefit of additional chemotherapy occurring in subjects with stage III disease or serous cancer. ${ }^{16}$

In a subgroup analysis, subjects with stage III disease demonstrated the greatest absolute benefit from the combined treatment, with $69.3 \%$ of patients in the chemoradiotherapy arm alive and recurrence-free at 5 years compared with $58 \%$ in the radiotherapy-only arm ( $\mathrm{HR} \mathrm{0.66,95 \%} \mathrm{Cl} \mathrm{0.45-}$ 0.97, adjusted $p=0.014) .{ }^{15}$ These patients are at higher risk of recurrence than patients with stage I or || disease. However, women with lower-stage disease also demonstrated benefit from the combined therapy, albeit to a lesser degree. Pelvic recurrence was also decreased. At 5 years, pelvic recurrence was $4.9 \%$ in the chemoradiotherapy group compared with $9.2 \%$ in the radiotherapy-only group $(p=0.026)$, confirming the importance of combination therapy to maximize local control of recurrence. While significantly more grade $\geq 3$ adverse events were reported in the chemoradiotherapy group compared with radiotherapy-only (60\% versus $12 \%)$, this difference vanished after the first 12 months of follow-up.

Given the differences in the trial designs of GOG 258 and PORTEC-3, the optimal adjuvant treatment of women with advanced-stage highrisk endometrial cancer remains unclear. The evidence to support combination chemoradiotherapy is strongest for women with stage III disease or serous histology, based on the findings of the PORTEC-3 trial, while GOG 258 supports the primacy of systemic adjuvant therapy for women with advanced endometrial cancer.

\section{Novel therapies and future directions}

Besides clinical and histopathological features, molecular features have validated prognostic value in endometrial cancer. An integrated genomics analysis by The Cancer Genome Atlas (TCGA) project proposed four molecular cohorts of endometrial cancer defined by their somatic mutational burden and copy number alterations. ${ }^{17}$ These cohorts include ultra-mutated endometrial cancer with mutations in DNA polymerase epsilon (POLE), hypermutated endometrial cancer with microsatellite instability (MSI), copy-number-high endometrial cancer with frequent TP53 mutations ("serous-like"), and copy-number-low endometrial cancers. Further research is needed to understand the ways in which these molecular classifications may be used to help guide therapy. For example, despite their ultra-mutated status, POLE-mutated endometrial cancers are associated with a favourable prognosis. This was affirmed in PORTEC-1 but appears unrelated to any heightened sensitivity to adjuvant therapies based on in vitro cell line models, ${ }^{18}$ and may instead represent enhanced immunogenicity. ${ }^{19,20}$

The ongoing PORTEC-4a trial will use a risk profile integrating TCGA molecular subgroups with L1-CAM overexpression, substantial lymphovascular space invasion, and CTNNB1-exon 3 mutations to triage patients with high-intermediate risk endometrial cancer to observation, vaginal brachytherapy, or pelvic radiation. ${ }^{21}$ The outcomes will be compared to a standard arm in which all high-intermediate risk patients receive vaginal brachytherapy.
The potential utility of immune checkpoint inhibitors in adjuvant therapy is also under investigation. Immune checkpoint therapy has demonstrated activity in advanced solid tumours, demonstrating mismatch repair (MMR) deficiency, MSI, or programmed death ligand 1 (PD-L1) expression. Immune checkpoint inhibitor therapy is becoming standard therapy in the treatment of recurrent and metastatic endometrial cancer previously treated with chemotherapy. In 2017, the US Food and Drug Administration (FDA) granted accelerated approval to pembrolizumab (anti-programmed cell death protein 1 [PD-1]) as the first tissue-agnostic therapy for treatment of unresectable or metastatic MSI-high or MMR deficient solid tumours with progression following prior treatment. The clinical studies on which this approval was based included 15 MMR-deficient endometrial cancers and demonstrated a $53 \%$ objective response rate (ORR) and a $74 \%$ disease control rate. ${ }^{22}$ An updated analysis demonstrated an ORR of $57 \%$ and median PFS of 25.7 months among 49 subjects with MMR-deficient recurrent endometrial cancer. ${ }^{23}$

More recently, pembrolizumab has been studied in combination with lenvantinib, a multikinase inhibitor, for the treatment of recurrent endometrial cancer, on the basis of preclinical studies indicating a synergistic effect of the two treatments. The FDA approved this combination therapy in $\mathbf{2 0 1 9}$ for the indication of recurrent endometrial cancer that is not MSI-high or MMR-deficient and in those who have disease progression following prior systemic therapy but are not candidates for curative surgery or radiation. The final results of the phase $\mathrm{Ib} / \mathrm{ll}$ trial are encouraging, with an overall ORR of $38 \%$ at 24 weeks. ${ }^{24}$ In subgroup analysis, the ORR was $63.6 \%$ in MSI-high tumours and $36.2 \%$ in microsatellite-stable tumours. ${ }^{24}$

Ongoing studies will help to further elucidate the role of immunotherapy in adjuvant treatment. One such study (ClinicalTrials.gov Identifier: NCT04214067) will compare radiation therapy with or without pembrolizumab in patients with newly diagnosed stage I-II highintermediate risk endometrioid endometrial cancer with demonstrated MMR deficiency, with 3-year PFS as the primary outcome..25 This study is currently in the recruitment phase and is anticipated to be completed in February 2024. Another study (ClinicalTrials.gov Identifier: NCT03914612) will compare carboplatin and paclitaxel chemotherapy with or without pembrolizumab in patients with stage III-IV and recurrent endometrial cancer and will examine 5-year PFS as its primary endpoint. ${ }^{26}$ This study is in the recruitment phase and is anticipated to be completed in June 2023. Other interesting potential strategies include targeting aberrant cell surface receptors or hyperactive signalling pathways, DNA damage repair deficits, and hormonal/metabolic dependencies and epigenetic reprogramming.

\section{Conclusions}

Overall, more research is needed to elucidate the optimal management of high-risk endometrial cancers and to decrease recurrence, minimize toxicity, and improve survival. The recently completed clinical trials reviewed herein have established evidence-based standards and benchmarks for designing the endometrial cancer trials of the future. While there are a number of unanswered questions regarding the optimal application of chemotherapy, radiotherapy and combination treatments tailored to individualized risks of local and distant recurrence, it is critical to leverage the knowledge gained through advances in the understanding of the genetic and molecular characteristics of endometrial cancer to guide the development of innovative adjuvant therapy approaches. Promising strategies include targeted therapies to exploit cancer vulnerabilities and dependencies, and engagement of the immune 
system. Currently enrolling studies will provide important new data on the utility of immunotherapy and other targeted therapies in the adjuvant setting. Going forward, molecular classification opens the possibility to deploy conventional and novel therapeutics to those patients most likely to benefit, while avoiding unnecessary toxicity in others. We anticipate the results of these ongoing and future trials to transform the landscape of adjuvant therapy, and ultimately, to significantly reduce endometrial cancer morbidity and mortality. $\square$
1. Siegel RL, Miller KD, Jemal A. Cancer statistics, 2020. CA Cancer J Clin. 2020;70:7-30

2. Miller KD, Nogueira L, Mariotto $A B$, et al. Cancer treatment and survivorship statistics, 2019. CA Cancer J Clin. 2019;69:363-85. Siegel RL, Miller KD, Jemal A. Cancer statistics, 2019. CA Cancer Siegel RL, Miller KD,
J Clin. 2019;69:7-34.

4. Bokhman JV. Two pathogenetic types of endometrial carcinoma. Gynecol Oncol. 1983;15:10-7.

5. Brooks RA, Fleming GF, Lastra RR, et al. Curren recommendations and recent progress in endometrial cancer. CA Cancer J Clin. 2019;69:258-79.

6. Keys HM, Roberts JA, Brunetto VL, et al. A phase III trial of surgery with or without adjunctive external pelvic radiation therapy in intermediate risk endometrial adenocarcinoma: a Gynecologic Oncology Group study. Gynecol Oncol. 2004;92:744-51.

7. Creutzberg $C L$, van Putten WL, Koper PC, et al. Surgery and postoperative radiotherapy versus surgery alone for patients with stage-1 endometrial carcinoma: multicentre randomised trial. Lancet. 2000;355:1404-11.

8. van den Heerik AS, Horeweg N, de Boer SM, et al. Adjuvant therapy for endometrial cancer in the era of molecular classification: radiotherapy, chemoradiation, and novel targets for therapy. radiotherapy, chemoradiation, and novel
Int J Gynecol Cancer. 2021;31:594-604.

9. Nout RA, Smit VT, Putter H, et al. Vaginal brachytherapy versus pelvic external beam radiotherapy for patients with endometrial cancer of high-intermediate risk (PORTEC-2): an open-label, non-inferiority, randomised trial. Lancet. 2010;375:816-23.

10. Randall ME, Filiaci V, MCMeekin DS, et al. Phase III trial: adjuvant pelvic radiation therapy versus vaginal brachytherapy plus paclitaxel/carboplatin in high-intermediate and high-risk earlystage endometrial cancer. I Clin Oncol. 2019:37:1810-18.

11. Randall ME, Filiaci VL, Muss H, et al. Randomized phase trial of whole-abdominal irradiation versus doxorubicin and cisplatin chemotherapy in advanced endometrial carcinoma: a Gynecologic Oncology Group study. J Clin Oncol. 2006;24:36-44.

12. Greven $\mathrm{K}$, Winter $\mathrm{K}$, Underhill $\mathrm{K}$, et al. Final analysis of RTOG 9708: adjuvant postoperative irradiation combined with cisplatin/paclitaxel chemotherapy following surgery for patients with high-risk endometrial cancer. Gynecol Oncol. 2006;103:155-9.

13. Homesley HD, Filiaci V, Gibbons SK, et al. A randomized phase III trial in advanced endometrial carcinoma of surgery and volume directed radiation followed by cisplatin and doxorubicin with or without paclitaxel: a Gynecologic Oncology Group study. Gynecol Oncol. 2008;112:543-52.

14. Matei D, Filiaci V, Randall ME, et al. Adjuvant chemotherapy plus radiation for locally advanced endometrial cancer. N Engl J Med. 2019;380:2317-26.

15. de Boer SM, Powell ME, Mileshkin L, et al. Adjuvant chemotherapy versus radiotherapy alone for women with high-risk endometrial cancer (PORTEC-3): final results of an inghisk enter international, open-label, multicentre,

16. de Boer SM, Powell ME, Mileshkin L, et al. Adjuvant chemotherapy versus radiotherapy alone in women with highrisk endometrial cancer (PORTEC-3): patterns of recurrence an post-hoc survival analysis of a randomized phase 3 trial. Lancet Oncol. 2019;20:1273-85.

17. Kandoth C, Schultz N, Cherniack AD, et al. Integrated genomic characterization of endometrial carcinoma. Nature. 2013;497:67-73.

18. Van Gool IC, Rayner E, Osse EM, et al. Adjuvant treatment for POLE proofreading domain-mutant cancers: sensitivity to radiotherapy, chemotherapy, and nucleoside analogues. Clin Cancer Res. 2018:24:3197-203.

19. Bellone S, Bignotti E, Lonardi S, et al. Polymerase E (POLE) ultra-mutation in uterine tumors correlates with T lymphocyte infiltration and increased resistance to platinum-based chemotherapy in vitro. Gynecol Oncol. 2017;144:146-52

20. Santin AD, Bellone S, Buza N, et al. Regression of chemotherapy-resistant polymerase $\mathrm{E}$ (POLE) ultra-mutated and MSH6 hyper-mutated endometrial tumors with nivolumab. Clin Cancer Res. 2016;22:5682-7.

21. van den Heerik ASVM, Horeweg N, Nout RA, et al. PORTEC-4 international randomized trial of molecular profile-based adjuvant treatment for women with high-intermediate risk endometrial cancer. Int J Gynecol Cancer. 2020;30:2002-7.

22. Le DT, Durham JN, Smith KN, et al. Mismatch repair deficiency predicts response of solid tumors to PD-1 blockade. Science. 2017;257:409-13.

23. Marabelle A, Le DT, Ascierto PA, et al. Efficacy of pembrolizumab in patients with noncolorectal hig microsatellite instability/mismatch repair-deficient cancer results from the phase II KEYNOTE-158 study. J Clin Oncol. 2019;38:1-10.

24. Makker V, Taylor MH, Aghajanian C, et al. Lenvatinib plus pembrolizumab in patients with advanced endometrial cancer J Clin Oncol. 2020;38:2981-92.

25. US National Library of Medicine. Testing the addition of the immunotherapy drug, pembrolizumab, to the usual radiation treatment for newly diagnosed early stage high intermediate risk endometrial cancer. Last updated 23 February 2021 Available at: https://clinicaltrials.gov/ct2/show/NCT04214067 (accessed 19 February 2021).

26. US National Library of Medicine. Testing the addition of the immunotherapy drug pembrolizumab to the usual chemotherapy treatment (paclitaxel and carboplatin) in stage III-IV or recurrent endometrial cancer. Last updated 23 February 2012. Available at: https://clinicaltrials. gov/ct2/show/ NCT03914612 (accessed 19 February 2021). 